\title{
Investigation of Hydrodynamic Flow Characteristics in Helical Coils with Ovality and Wrinkles
}

\author{
Govindaraj Periasamy1, ${ }^{*}$ - Senthilkumar Mouleeswaran ${ }^{2}$ - Prabhu Raja Venugopal ${ }^{1}$ - Chellapandi Perumal $^{3}$ \\ ${ }^{1}$ PSG College of Technology, Department of Mechanical Engineering, India \\ 2 PSG College of Technology, Department of Production Engineering, India \\ ${ }^{3}$ Indian Institute of Technology Madras, Department of Applied Mechanics, India
}

The forming of helical coils using a rolling process results in geometrical irregularities (wrinkles and ovality) that are likely to influence the hydrodynamic behaviour of the flow field inside the coil in applications such as air generators. In this study, the above behaviour was investigated by experimental and numerical analyses considering the heat exchanger used in dry air generators. In experimental analysis, a three-turn copper helical coil with wrinkles and ovality was investigated to estimate the global hydrodynamic characteristics inside the helical coil. The results were compared with that of the ideal geometry of a coil without wrinkles and ovality. The effect of wrinkles was assessed through friction factor, and the corresponding equivalent surface roughness was found to increase by 5.7 times, owing to the presence of wrinkles in the helical coil. Numerical simulation was conducted to determine the pressure distribution, velocity distribution, and secondary flow inside the helical coil; the results were validated with experimental data. A critical portion of the helical coil with multiple wrinkles was considered for numerical simulation to investigate the localized effects of wrinkles on the flow field behaviour. The analysis in the vicinity of wrinkles revealed negative pressure development during flow, which in turn would cause re-circulation and cavitation that are undesirable.

Keywords: helical coil, flow characteristics, ovality, wrinkles, computational fluid dynamics
Highlights
- The effect of wrinkles and ovality on the flow field in a copper helical coil was investigated by experimental and numerical analysis.
- $\quad$ The maximum ovality in a helical coil was found to be $3.2 \%$, and it does not significantly affect the hydrodynamic characteristics of the flow field.
- $\quad$ The wrinkles contribute significantly to the pressure drop in the helical coil; the maximum pressure drop was doubled due to the presence of wrinkles when the Reynolds number (Re) attains 100,000.
- The effect of wrinkles was assessed through the friction factor, and the corresponding equivalent surface roughness was found to increase by 5.7 times owing to the presence of wrinkles in helical coils.
- $\quad$ The numerical analysis of the critical portion of the helical coil exhibited local damage mechanisms, such as cavitation due to negative pressure developed in the vicinity of wrinkles.

\section{INTRODUCTION}

Helical coils are extensively used in several industries in various applications due to their compact size and ability to accommodate thermal expansion at elevated temperatures. The fluid flowing through the helical coil experiences a centrifugal force due to the curvature effect and leads to secondary flow perpendicular to the axial flow of the fluid. Due to secondary flow, the fluid particles move towards the outer wall and retract back to the inner wall [1]. The torsion effect in the helical coil along with secondary flow leads to increased pressure drop and makes the flow more complex. Dean [2] presented the correlations for flow in curved tubes and defined the Dean effect associated with curvature. The geometric parameters of the helical coil (i.e., helical pitch, pitch circle diameter (PCD), number of helical turns, and secondary flow) highly influence the flow behaviour of fluid inside the helical coil [3]. The effect of geometry and inlet conditions is vital in predicting the mass flow rate for the given Reynolds number [4]. The secondary flow characteristics and their influence on the pressure drop in a helical coil depend on geometrical parameters [5]. The different flow configurations of the coil will significantly affect the performance of coils [6]. Fluid flow and heat transfer characteristics in spiral coils are interrelated [7]. The above parameters also influence the heat transfer rate of tube-in-tube helical coils [8]. The heat transfer behaviour in the laminar and turbulent conditions of tube-in-tube helical coils can be characterized by the local Nusselt number [9]. The heat transfer rates in helical coils are more dependent on the geometrical parameters when compared with straight tubes [10]. The heat transfer in heat exchangers with dimple patterns will depend on the geometry of the dimple pattern; therefore, it is important to analyse the influence of wrinkles in coils [11]. The helical double pipe heat exchanger will have both geometrical and flow complexities owing to 
its flow behaviour. It is important to understand the hydrodynamics of the fluid inside the coil to enhance heat transfer rate [12] and [13].

Tube-in-tube co-axial helical coils are widely used as heat exchangers in refrigeration air dryer systems as pre-coolers and evaporators to remove moisture content from compressed air. To maximize the heat transfer rate, copper is selected as the tube material. Copper tubes of lengths of 5 to 7 metres are manufactured and rolled into tube-in-tube helical coils of required pitch circle diameter using a three-roller tube bending machine. During the transportation of straight tubes, minor dimples form in the copper tubes. While rolling the lengthy tubes into compact helical coils, wrinkles form at the intrados of the tubes. Thinning and thickening take place at extrados and intrados of the helical tube, respectively, and result in flattening of helical coil. The resulting geometrical irregularities, namely, ovality and wrinkles, obstruct the flow of fluid and create turbulence during fluid flow, which results in pressure drop during operation. The pressure drop affects the effectiveness of heat transfer and results in insufficient dry air generation [14]. Based on an exhaustive literature review, it was found that limited work has been done on the influence of geometrical irregularities on the flow behaviour in helical coils. Hence, the present work aims to investigate the implications of wrinkles and ovality on the flow fields in helical coils, taking into consideration the flow velocity variations.

\section{MEASUREMENT OF OVALITY AND SYNTHESIS OF WRINKLES IN HELICAL COILS}

The helical coils formed during bending have two major geometrical irregularities: ovality and wrinkles. The ovality or flattening of a bend is defined as the difference between the maximum and minimum outside diameters at any cross-section, expressed as a percentage of the nominal outside diameter. The heat exchanger used in a dry air generator is considered for the present study. It consists of tube-in-tube copper helical coils, formed using a three-roller pipe bending machine, as shown in Fig. 1.

A three-turn helical coil used in the tube-in-tube co-axial heat exchanger for the selected industrial application is taken up for investigation. Further, only the outer coil $(D=50.08 \mathrm{~mm})$ without the inner coil ( $d=15 \mathrm{~mm})$ is considered for study since the outer coil, with the larger coil diameter, is susceptible to higher wrinkle formation during pipe bending. To fabricate the outer helical coil, a long tube of outer diameter $50.08 \mathrm{~mm}$, thickness $1 \mathrm{~mm}$, and the developed length of $5 \mathrm{~m}$ was passed between the upper and lower rollers. The three-turn coil was formed in three stages, by passing the copper coil between rollers and gradually increasing the feed to achieve the intended pitch circle diameter of $650 \mathrm{~mm}$ and pitch of $52 \mathrm{~mm}$.

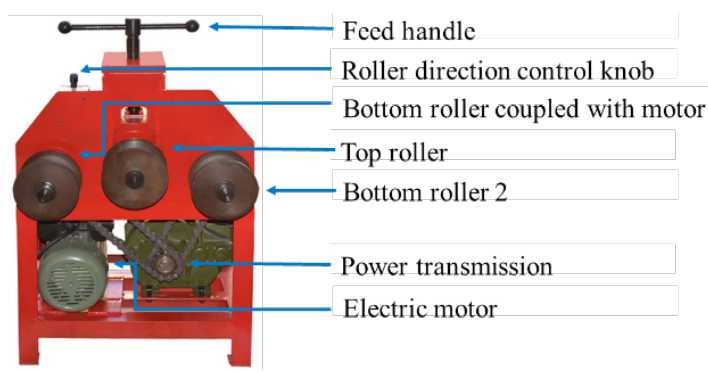

Fig. 1. Three roller tube bending machine

As per ASME B31.1 (N-129.1.1) standard [15], ovality shall not exceed $8 \%$ for tubes exposed to internal pressure. For tubes subjected to external pressure, ovality shall not exceed $3 \%$.

$$
\text { Percentage ovality }=\frac{D_{\max }-D_{\min }}{D} \times 100 .
$$

The relation for calculating percentage ovality is given in Eq. (1), where $D_{\max }$ and $D_{\min }$ represent the major and minor diameters of the geometrical irregularity in the form of the ovality, as shown in Fig. 2.

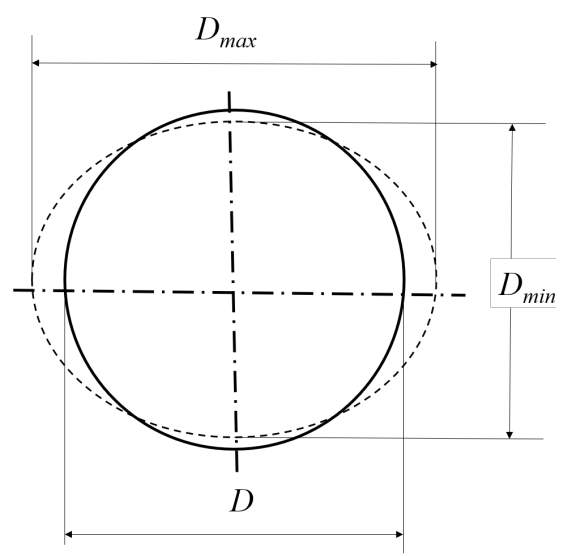

Fig. 2. Geometric representation of ovality

Considering several helical coils for measuring ovality, the maximum ovality was $3.2 \%$ which is well within the allowable limits as per ASME B31.3 code. Hence, ovality is not likely to influence the flow behaviour.

The other geometrical irregularity, wrinkles, occurs only at the intrados due to compression while 
forming helical coils. Considering a critical portion of a sample coil for a length of $300 \mathrm{~mm}$, there were 24 wrinkles of different sizes, as represented in Fig. 3.

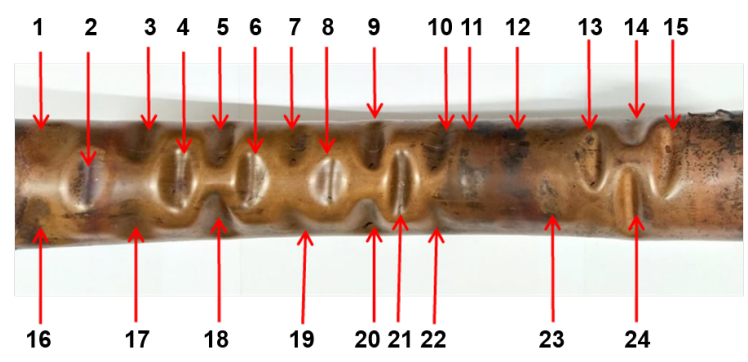

Fig. 3. Wrinkles in critical portion of helical coil

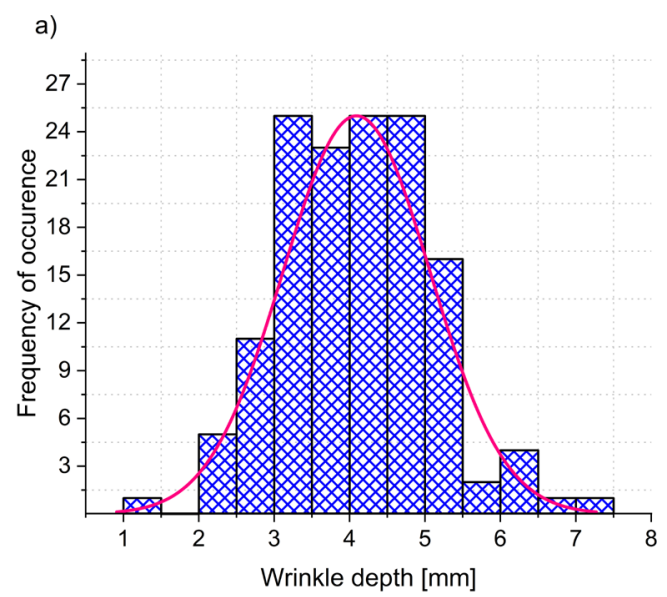

b)

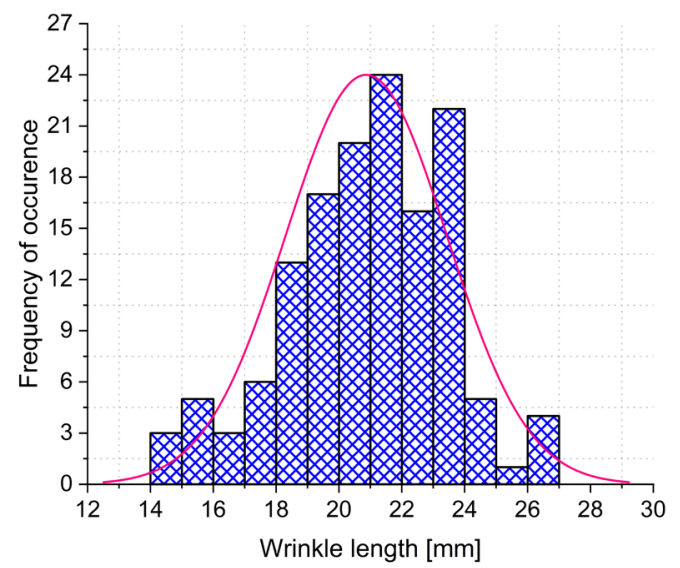

Fig. 4. Probability distribution of wrinkles; a) $\mu=4.07, \sigma=1.39$, and b) $\mu=20.89, \sigma=3.75$

Considering the total length of $5 \mathrm{~m}$ of another full coil, 139 wrinkles of varying size were measured, and their distribution is obtained by employing Gaussian fit using MATLAB ${ }^{\circledR}$. Fig. 4 shows the frequency of occurrence of wrinkles in terms of depth and length, and the histogram reveals a normal distribution.
Among the wrinkles, the maximum depth and length were $6.8 \mathrm{~mm}$ and $32 \mathrm{~mm}$, respectively. Subsequently, the effect of wrinkles on the hydrodynamic flow characteristics in the helical coil is investigated.

The measured pressure drop will be used to analytically determine the friction factor and subsequently the equivalent surface roughness of the coil.

\section{ASSESSMENT OF THE EFFECT OF WRINKLES ON EQUIVALENT SURFACE ROUGHNESS OF HELICAL COIL}

\subsection{Measurement of Pressure Drop}

A schematic representation of the experimental set up is shown in Fig. 5. The set-up contains the following components: a copper helical tube, centrifugal pump, flow meter, pressure gauges, valves, and tube lines. Compressed air at room temperature was pumped to a storage tank and allowed to flow through the pneumatic circuit. As the coil was mounted vertically, it resulted in a downstream flow of air. Since the coil being investigated is meant for use in a dry air generator, a wider range of operating flow conditions needs to be considered. Hence, a valve was used to regulate the flow. The mass flow rate was measured using the differential pressure flow measurement principle. Pressure gauges were provided to measure the operating pressure at salient points in the circuit.

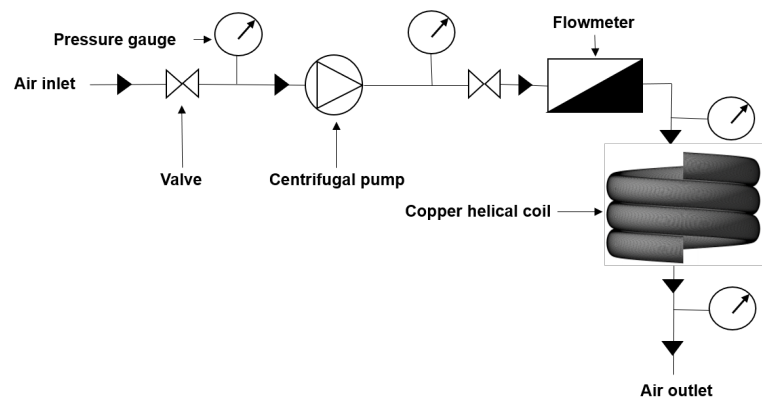

Fig. 5. Schematic diagram of experimental set-up

The flow rate was varied from $34 \mathrm{~m}^{3} / \mathrm{h}$ to 850 $\mathrm{m}^{3} / \mathrm{h}$ in steps with corresponding pressures varying from 7 bar to 16 bar. The pressure drop in the coil was obtained by measuring the air pressures at the inlet and outlet of the coil. The global $R_{e}$ was calculated experimentally based on the velocity of compressed air inside the coil using Eq. (2).

$$
R_{e}=\frac{\rho V D}{\mu},
$$


where $\rho, V, D$ and $\mu$ are density of air $\left[\mathrm{kg} / \mathrm{m}^{3}\right]$, average flow velocity of air $[\mathrm{m} / \mathrm{s}]$, coil diameter $[\mathrm{m}]$ and dynamic viscosity of air $[\mathrm{kg} /(\mathrm{ms})]$ respectively. Three trials of experiments were carried out for every operating condition. The variations in pressure drop with increase in $R_{e}$ were investigated by considering coils, with and without wrinkles, and a comparison is shown in Fig. 6.

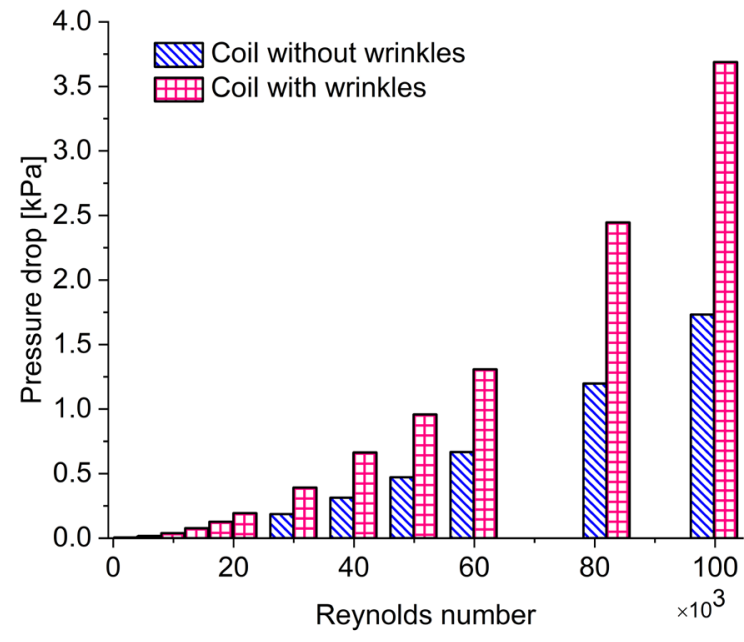

Fig. 6. Pressure drop based on experimental results

It is observed that wrinkles contribute significantly to pressure drop in the helical coil. A peak pressure difference of $2 \mathrm{kPa}$ was observed when $R_{e}$ of 100,000 was attained. A smooth pipe with wrinkles and ovality behaves as a rough pipe [16]. Hence, the friction factor is calculated from the experimental value of pressure drop for a specific $R_{e}$ in order to arrive at an equivalent surface roughness of the coil.

\subsection{Determination of Friction Factor and Equivalent Surface Roughness of Helical Coil}

The equivalent surface roughness is the roughness of a non-wrinkled coil whose friction factor is equal to that of a wrinkled coil. In order to assess the effect of wrinkles, the fanning friction factor [17], which contributes to the pressure drop, is calculated from,

$$
\Delta P=\frac{2 f_{c} \rho u^{2} L}{D},
$$

where $u, f_{c}, \Delta P, \rho, D$ and $L$ are average flow velocity of air $[\mathrm{m} / \mathrm{s}]$, fanning friction factor, pressure drop [Pa], density of air $\left[\mathrm{kg} / \mathrm{m}^{3}\right]$, coil diameter $[\mathrm{m}]$, coil length [m] respectively. The effect of wrinkles and $R_{e}$ on friction factor is shown in Fig. 7.
The friction factor increases by $78 \%$ at the lowest $R_{e}$ owing to the presence of wrinkles, which will have higher implications on the flow field. The friction factor is independent of $R_{e}$ beyond 60,000 and it is found to be 0.010 and 0.018 for coil without wrinkles and coil with wrinkles, respectively.

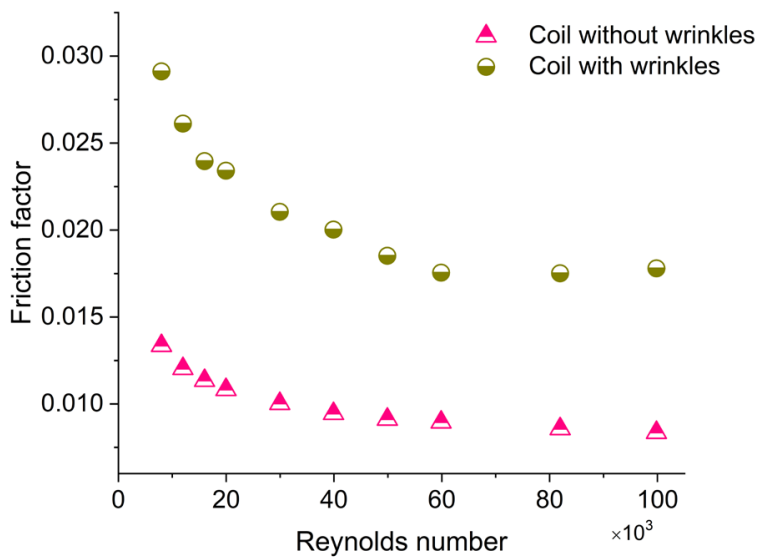

Fig. 7. Friction factor of coils

The widely accepted Colebrook [18] formula (Eq. (4)) gives a relation between the friction factor, $R_{e}$ and equivalent surface roughness $(\varepsilon)$, for $R_{e}$ above 4000 . It is used to arrive at $\varepsilon$, given the friction factor for a specific Reynolds number [19].

$$
\frac{1}{\sqrt{f}}=-4 \log \left[\frac{\varepsilon}{3.7 D}+\frac{1.256}{R_{e} \sqrt{f}}\right] .
$$

Initially, the surface roughness of the coil at the inner wall, in no flow condition, was measured using a portable surface roughness tester (SURFTEST SJ $41{ }^{\circledR}$ series), as shown in Fig. 8. Five trials were taken at each section with a cut-off range of $0.25 \mathrm{~mm}$ and $0.08 \mathrm{~mm}$, and the roughness values were found to be $0.747,0.181,2.075,1.100$ and 1.470 . The average surface roughness of $1.11 \mu \mathrm{m}$ is taken as the reference as shown as a horizontal line in Fig. 9.

It is found from the figure that the maximum $\varepsilon$ of $6.17 \mu \mathrm{m}$ is 5.7 times more than that of the commercially available tubes. which is attributed to the combined influence of wrinkles and flow at lowest $R_{e}$. It is also found that the roughness of coil with wrinkles and that without wrinkles is $2.3 \mu \mathrm{m}$ and $0.3 \mu \mathrm{m}$ beyond $R_{e}$ of 60,000 . At the lowest $R_{e}$, the equivalent surface roughness of the coil will cause a significant pressure drop (Fig. 6) and will affect the overall heat transfer performance of the coil.

When $R_{e}$ is 10,000 , the copper coil is found to behave like a rough pipe without wrinkles. To visualize and better understand the effect of wrinkles 
on the performance of helical coil, a numerical investigation is carried out.

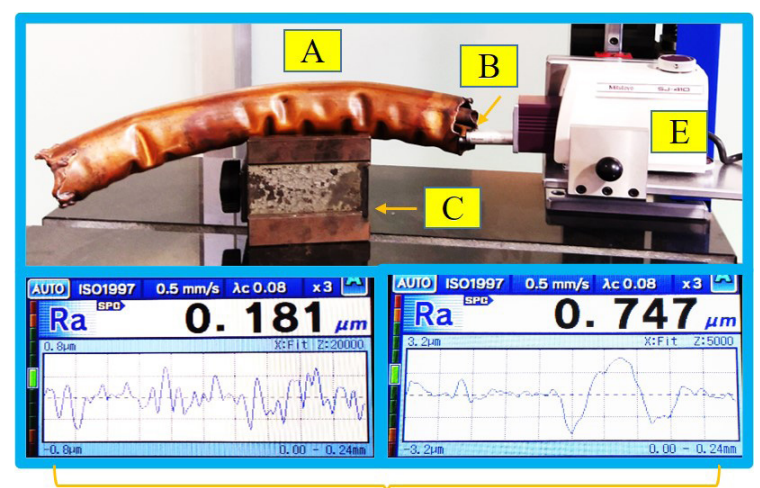

A - Helical coil

D

B - Inner wall surface to be tested

$\mathrm{C}-\mathrm{V}$ block to hold helical coil

D - Sample trial measurements

E - Portable surface roughness tester

Fig. 8. Surface roughness measurement

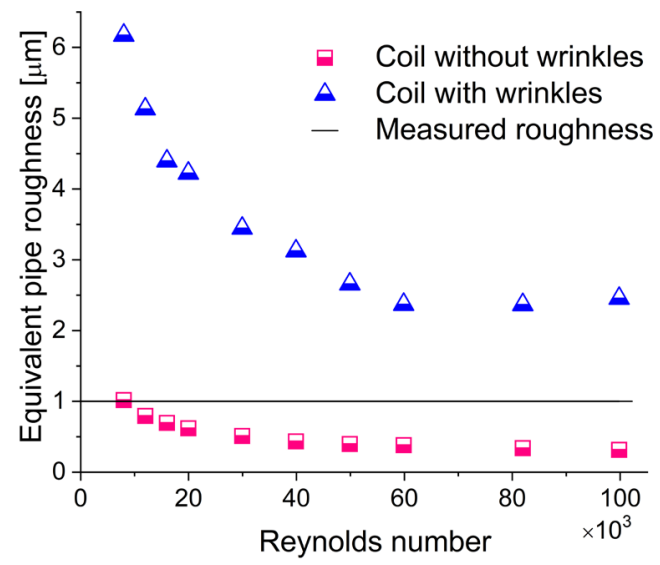

Fig. 9. Equivalent surface roughness of coil

\section{NUMERICAL INVESTIGATIONS ON THE IMPLICATIONS OF WRINKLES AND OVALITY ON THE FLOW FIELD IN HELICAL COIL}

The numerical investigation is conducted in two stages: (i) global analysis of three-turn coil with and without geometrical irregularities and (ii) local analysis of flow field in a critical portion of coil with wrinkles. Prior to investigating the effect of wrinkles and ovality on flow behaviour, an idealized coil without any geometrical irregularities was considered for analysis. The results will be used as a benchmark while investigating the fluid flow behaviour in the coil with wrinkles and ovality. Owing to the vertical orientation of coils, downstream flow is considered for analysis.

\subsection{Global Analysis of Hydrodynamic Behaviour of Fluid Field in Helical Coil}

For global analysis, the helical coil without wrinkles was modelled using $\mathrm{Creo}^{\circledR}$ and the model was imported into computational fluid dynamics (CFD) module of ANSYS ${ }^{\circledR}$ software. The boundary conditions that are applied on the model of an ideal coil are shown in Fig. 10.

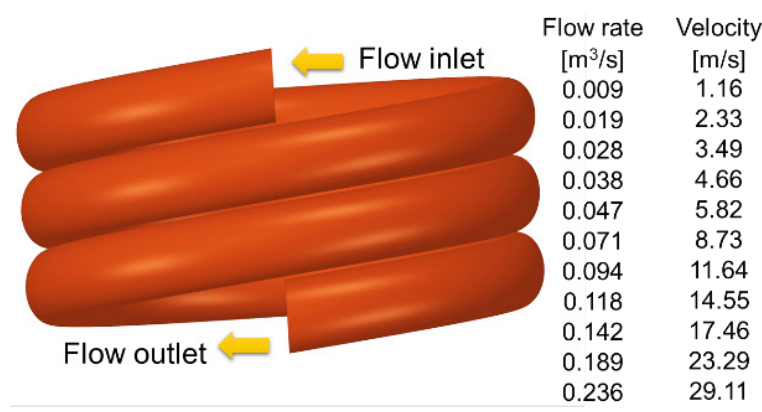

Fig. 10. Geometry and operating velocity range

A steady-state simulation was carried out by solving governing equations in a stationary framework. Air at $25{ }^{\circ} \mathrm{C}$ was used as the working fluid, and copper was assigned as the solid medium for the simulation of both models (i.e., ideal coil and wrinkled coil). The coil selected for analysis is designed to handle flow of air in the range of $0.009 \mathrm{~m}^{3} / \mathrm{s}$ to $850 \mathrm{~m}^{3} / \mathrm{s}$ and the corresponding flow velocity is ranging from $1.16 \mathrm{~m} / \mathrm{s}$ to $29.11 \mathrm{~m} / \mathrm{s}$. The "velocity inlet" condition is defined, and it is varied using the inlet "parameter set" option for simulation. The present investigation is mainly focused on the hydrodynamic behaviour of coils with geometrical deviation; hence, the outlet of coil is specified as the "open" condition. The scale and quality of the mesh were verified, and the solver type was selected as a velocity-based formulation considering downstream flow. Four turbulence models were used to perform simulation prior to arriving at a suitable model. The selected SST $k-\varepsilon$ model was found to be better at predicting flow characteristics in a helical coil than other models were; Piazza and Ciofalo [20] had similar findings. The solution method was specified as pressure-velocity coupling by adopting the SIMPLE scheme for solving the governing equation with a second-order upwind scheme. 


\subsubsection{Mesh Convergence Study}

A fine mesh was generated with a inflation layer for solid-fluid interfaces, and a grid independence study (Fig. 11) was performed by adequate edge sizing and inflation growth rates. Since mesh convergence was attained at point $\mathrm{D}$, the mesh corresponding to point $\mathrm{D}$ was used for further investigation. The same procedure was repeated for a coil with wrinkles, whose model was obtained by reverse engineering.

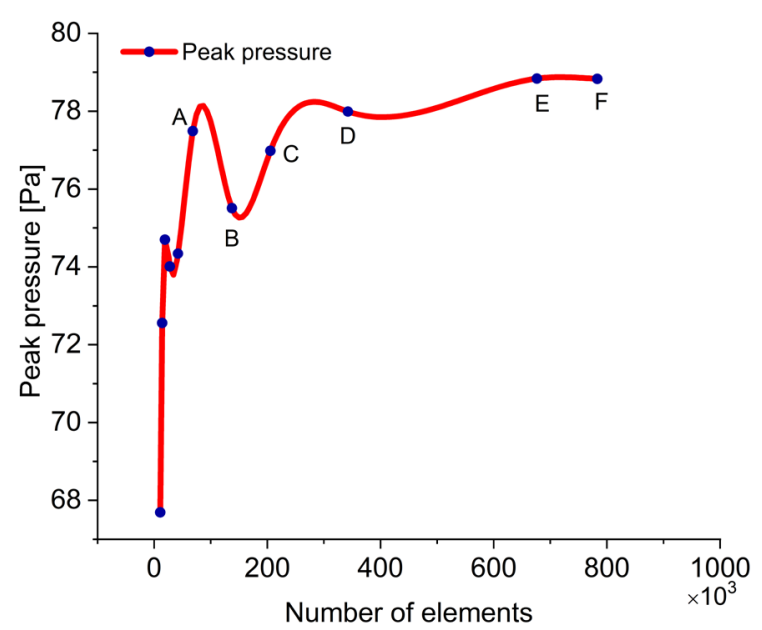

Fig. 11. Mesh sensitivity

The meshed model with inflation contains a combination of tetra and hexahedral elements to represent wrinkles of different sizes that have a combination of very steep to smooth surfaces. The adaptive meshing option aided in obtaining very fine meshing at the locations where wrinkles were present.

\subsubsection{Pressure Distributions at Different Angular Positions}

The pipe curvature causes centrifugal forces to act on the flowing fluid, resulting in a secondary flow pattern perpendicular to the main axial flow. Fig. 12 shows the pressure distribution $\left(R_{e}=49,000\right)$ along the outer wall, where secondary flow can be visualized at the extrados.

Up to an angle of $180^{\circ}$, secondary flow influences the pressure distribution and hence the pressure plots are maximum at the outer periphery of the coil; gradually, the pressure becomes uniform as the fluid approaches further turns of the helical coil. The helical coil was initially scanned using a blue light scanner (ATOS Compact Scan 2M) by placing stickers on the geometry, and the point cloud data was obtained to form a surface model. The above model was converted into a solid model using Geomagic Design $X^{\circledR}$ software (Fig. 13).

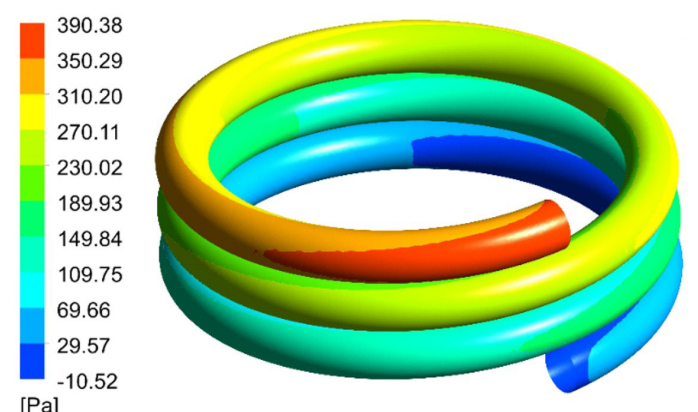

Fig. 12. Pressure distribution along the length in coil

a)

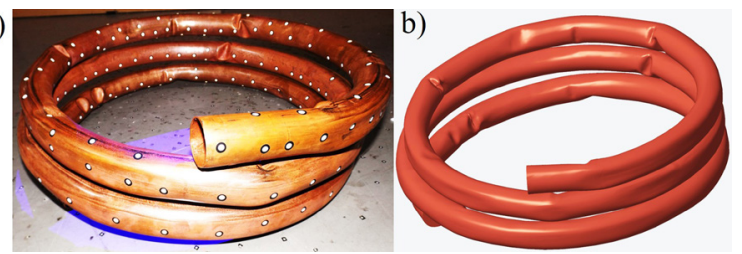

Fig. 13. Reverse engineering of wrinkled helical coil; a) reverse engineering, and b) extracted wrinkled coil

The model was used to perform finite element simulation after incorporating the same boundary conditions that were used for experimentation. The pressure distribution inside the wrinkled helical coil is shown in Fig. 14.

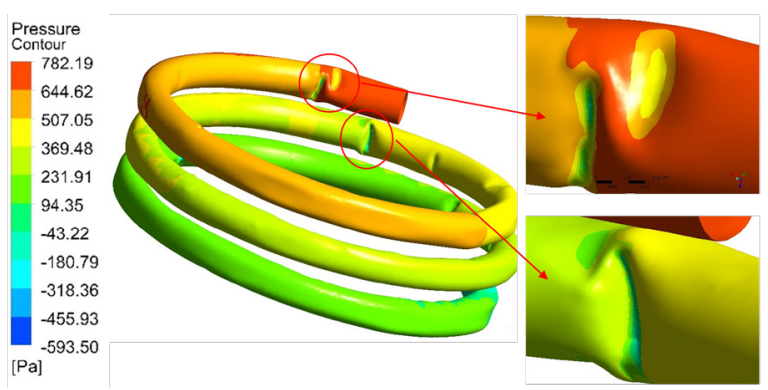

Fig. 14. Pressure distribution in wrinkled coil

It is found that there is a significant pressure drop in the wrinkled coil compared to the ideal coil, and there was considerable negative pressure developed in the wrinkled portions. This would lead to an increased velocity gradient in the vicinity of wrinkles and contribute to unsteady flow along the length of coil.

\subsubsection{Comparison of Streamline Patterns for Different Flow Parameters}

The surface velocity streamline at the vertical midplane of the helical coil is represented in Fig. 15. In 
a wrinkled coil, it can be observed that there is an unsteady surface streamline at the sections where wrinkles are present, and the shape imperfection has also influenced the formation of streamline in the corresponding sections of the plot. The maximum pressure in the wrinkled coil is found to be twice as that of the ideal coil without wrinkles.

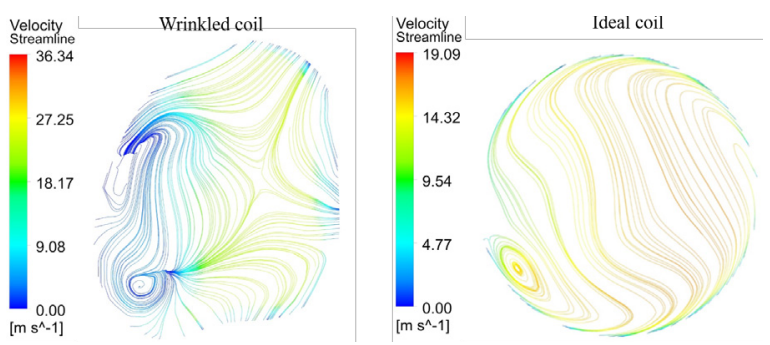

Fig. 15. Velocity streamline in wrinkled and ideal coils
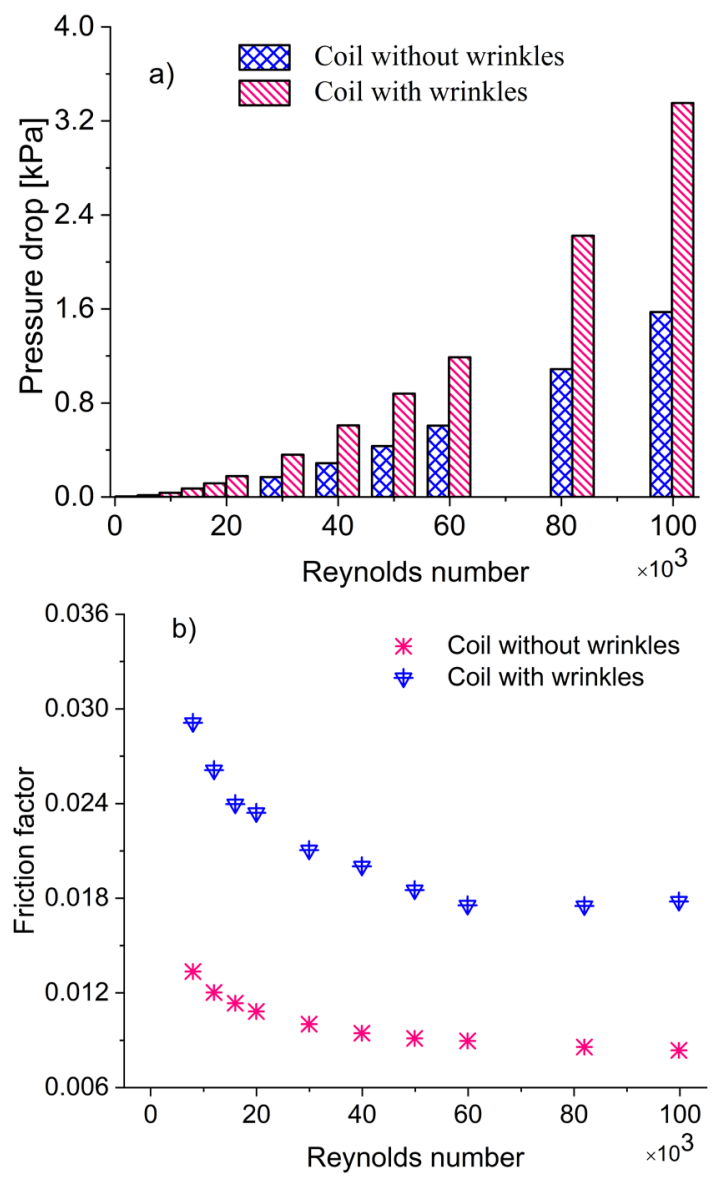

Fig. 16. Comparison of a) pressure drop, and b) friction factor based on numerical results

The parameters considered for numerical investigation are the same as those used for experimentation; a comparison of pressure drop and friction factor for various Reynolds numbers is shown in Fig. 16a and b, respectively.

It was found that the wrinkled coil experiences a two-fold higher pressure drop and friction factor when compared to a coil without wrinkles. It would significantly affect the overall pumping power and global heat transfer rate for tube-in-tube helical coil heat exchangers.

\subsubsection{Global Analysis of Velocity Variations along the Length of the Coil}

The behaviour of the flow field inside the helical coil was visualized using CFD software. The developed length of the coil of $5 \mathrm{~m}$ was considered for globally investigating the velocity along the length of the helical coil for a range of $R e$ from 3976 to 99,801 (Fig. 17).

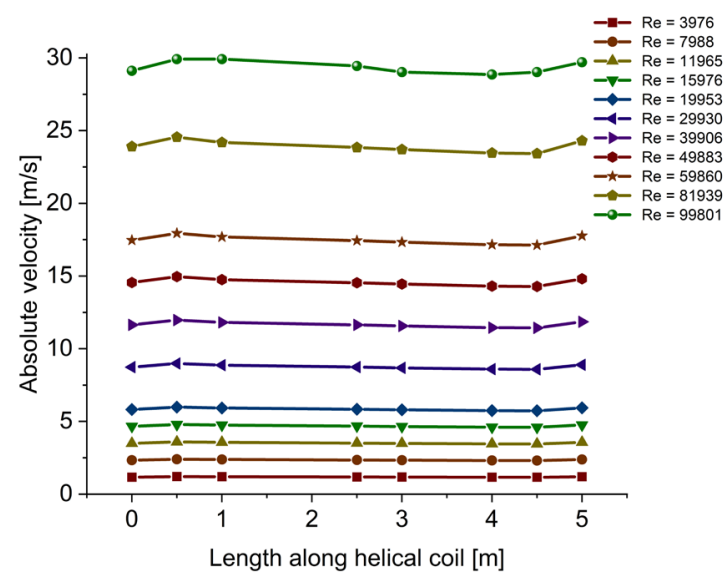

Fig. 17. Velocity fluctuation along the length of coil without wrinkles and ovality

The observed velocity profile remains almost constant for a specific Reynolds number. The velocity fluctuations are observed to be increasing to a considerable range beyond $R_{e}$ of 40,000 . The secondary flow due to helix angle and turbulence in the flow field are the primary reasons for velocity fluctuations in helical coils without wrinkles.

The flow velocities were measured at nine sections of the coil, as shown in Fig. 18. The absolute velocity of air along the length of the coil is found to be oscillating owing to the presence of wrinkles, which tend to reduce the cross-sectional area locally. By extracting the data on fluid velocity and crosssectional area from the simulation software, the discharge is found to be a constant with a magnitude of $0.057 \mathrm{~m}^{3} / \mathrm{s}$ and thus satisfying the continuity equation. 

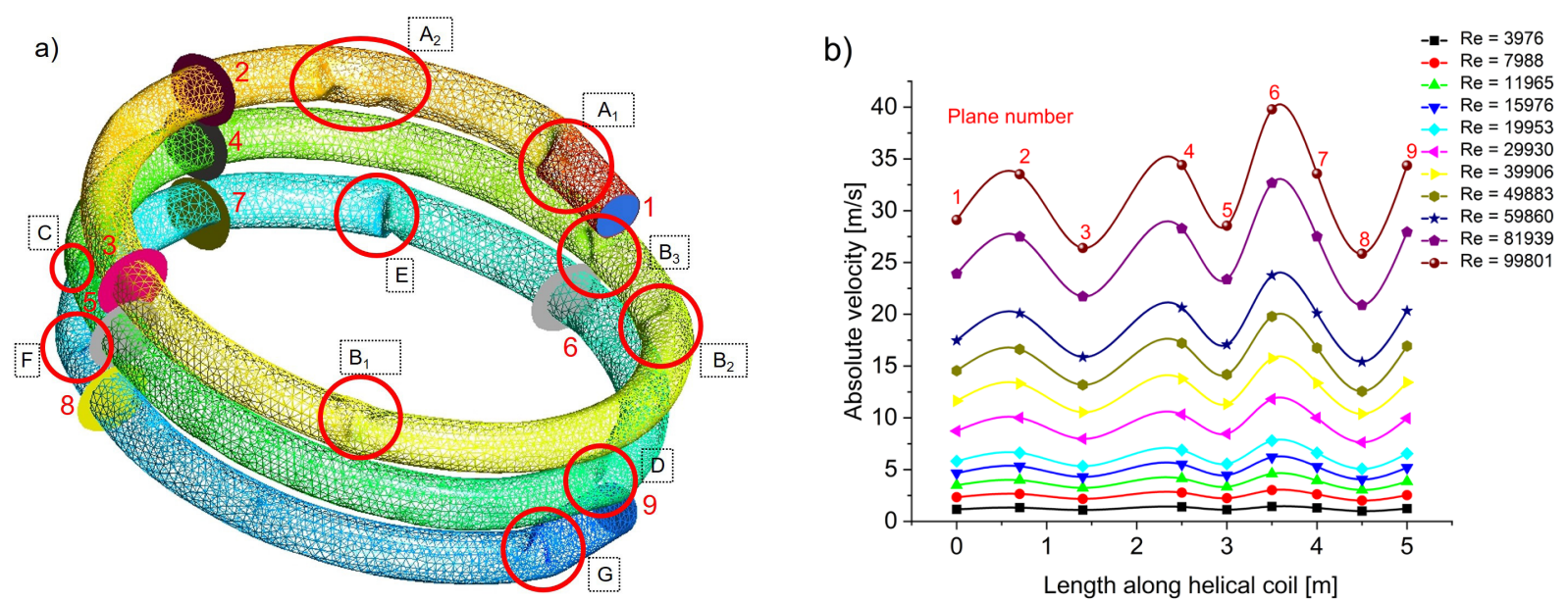

Fig. 18. a) Representation of critical wrinkles; and b) absolute velocity at selected sections

This implies that the sectional area owing to the presence of wrinkles could cause noticeable velocity variations cyclically along the length of the coil.

\subsubsection{Assessment of Influence of Ovality and Wrinkles on Flow Field through FRICTION FACTOR}

The maximum ovality of the helical coil was found to be $3.2 \%$ by measurement. The difference in friction factor between ideal coil and coil with ovality is 8.5 $\%$, whereas the difference in friction factor between ideal and wrinkled coils is $80.5 \%$. Hence, it is evident that ovality does not significantly influence the global behaviour of the flow field (Table 1).

Table 1. Comparison of friction factor

\begin{tabular}{llcc}
\hline $\begin{array}{l}\text { Geometry } \\
\text { (three turn helical coil) }\end{array}$ & \multicolumn{1}{c}{ Method } & $\begin{array}{c}\text { Pressure } \\
\text { drop [Pa] }\end{array}$ & $\begin{array}{c}\text { Friction } \\
\text { factor }\end{array}$ \\
\hline $\begin{array}{l}\text { Without ovality and } \\
\text { wrinkles }\end{array}$ & Numerical & 1293 & 0.0111 \\
\cline { 2 - 4 } & Experimental & 1303 & 0.0113 \\
\hline With ovality & Numerical & 1342 & 0.0121 \\
\hline $\begin{array}{l}\text { With ovality and } \\
\text { wrinkles }\end{array}$ & Numerical & 2036 & 0.0262 \\
\cline { 2 - 4 } & Experimental & 2132 & 0.0281 \\
\hline
\end{tabular}

The isolated wrinkles in the helical coil contribute to a significant global pressure drop and have a dominant effect on local behaviour, such as negative pressure. The above behaviour will affect the heat transfer aspects of a helical coil, leading to crack initiation. In the event of the fluid being a refrigerant, it will have a considerable environmental impact. Hence, it is necessary to investigate the local behaviour by considering a critical portion of the coil.
A much finer mesh is used for the critical portion to better capture the flow behaviour.

\subsection{Local Analysis of Flow Field in a Critical Portion of Helical Coil with Wrinkles}

A critically wrinkled portion of the coil is considered for numerical investigation, as already shown in Fig. 3. The geometric model for the critical portion of the coil was obtained using a reverse engineering approach. A finer mesh scheme was used to capture turbulence near the vicinity of wrinkles while developing the numerical model. The circumferential pressure distribution at a selected critical plane of the helical coil is shown in Fig. 19.

It can be observed that the pressure increases in all the cases near the extrados of the coil. The wrinkles increase the turbulence in the flow field and thereby causes higher secondary flow. This will result in flow loss due to increased wall shear stress. It can also be observed that the isolated wrinkles are not identical in shape and size; hence, the plots of circumferential pressure vary from each other.

\subsubsection{Effect of Negative Pressure in Wrinkled Coils}

To visualize the negative pressure zones, the positive pressure distribution along the coil was made to be $0 \mathrm{MPa}$, and negative pressure zones (Fig. 20) alone were concentrated.

The negative pressure formation is attributed to the coil's wrinkles and would create a vortex. The pressure and velocity distribution of air has been analysed. It is found that the local dents were 


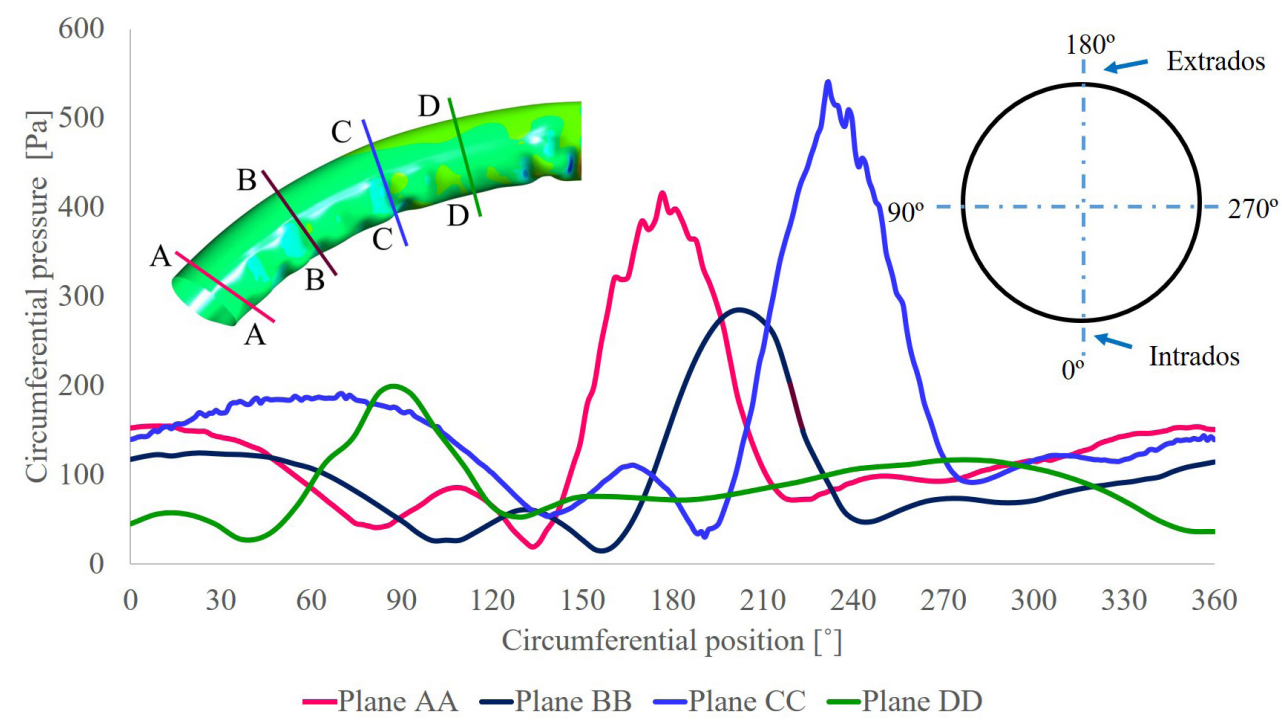

Fig. 19. Circumferential pressure distribution at critical sectional planes

isolated, which causes local re-circulation. At isolated locations, the occurrence of negative pressure may cause cavitation resulting in pitting.

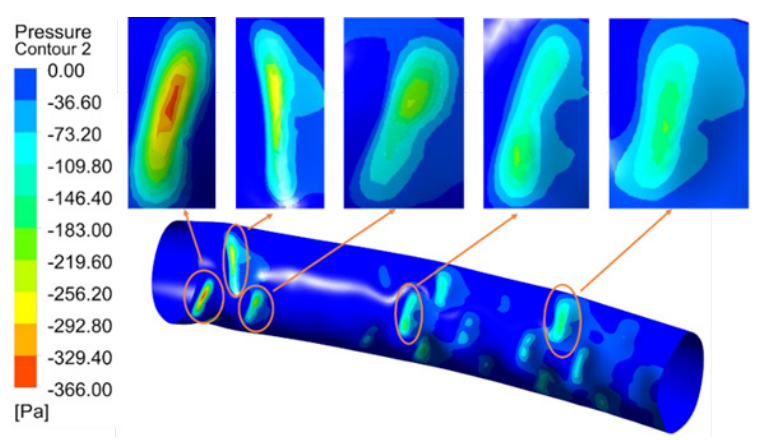

Fig. 20. Negative pressure formation

\section{CONCLUSIONS}

The major conclusions of the present work are as follows:

1. The numerical investigation revealed that the maximum ovality of $3.2 \%$ does not significantly influence the flow field in a helical coil.

2. The wrinkles are found to have significant effects on both the global and local flow behaviour. The global analysis of pressure drop and velocity field revealed the effect of wrinkles on the flow field in the form of a rise in equivalent roughness of the helical coil and oscillation of velocity distributions due to sectional area variations along the length of the helical coil.
3. The analysis of a critical portion of the helical coil revealed increased turbulence in the flow field. The resulting secondary flow has resulted in considerable negative pressure in the flow field, which would initiate localized damage mechanisms, such as cavitation and pitting. The above mechanisms would lead to more severe consequences when helical coils with wrinkles are used in heat exchangers for critical applications like nuclear, medical, etc.

4. Further investigation on material and structural damage is required to quantify heat transfer and other process effects in addition to the local negative pressure fields by considering the other geometrical irregularities, namely, thinning at extrados and thickening at the intrados.

5. The results of the present work are based on the investigation carried out for a particular size of coil; hence, it is valid only for the above configuration and for similar coils that have distributions of wrinkles as considered in the present work. Therefore, a parametric study that accounts for variations in coil geometry and associated wrinkle distribution and size is required.

\section{ACKNOWLEDGEMENTS}

We acknowledge PSG College of Technology for providing the necessary support required to carry out the work successfully. We also acknowledge M/s GEM Equipments Private Limited, Coimbatore, India 
for supporting us to conduct experiments in their facility.

\section{REFERENCES}

[1] Eustice, J. (1911). Experiments on stream-line motion in curved pipes. Proceedings of the Royal Society A: Mathematical, Physical and Engineering Sciences, vol. 85, no. 576, p. 119-131, Dol:10.1098/rspa.1911.0026.

[2] Dean, W.R. (1927). XVI. Note on the motion of fluid in a curved pipe. The London, Edinburgh, and Dublin Philosophical Magazine and Journal of Science, vol. 4, no. 20, p. 208-223, DOI:10.1080/14786440708564324.

[3] Jayakumar, J., Mahajani, S., Mandal, J., Vijayan, P., Bhoi, R. (2008). Experimental and CFD estimation of heat transfer in helically coiled heat exchangers. Chemical Engineering Research and Design, vol. 86, no. 3, p. 221-232, Dol:10.1016/j.cherd.2007.10.021.

[4] Dubba, S.K., Kumar, R. (2018). Experimental investigation on flow of r-600a inside a diabatic helically coiled capillary tube: Concentric configuration. International Journal of Refrigeration, vol. 86, p. 186-195, D0I:10.1016/j. ijrefrig.2017.10.035.

[5] Tang, L., Yuan, S., Malin, M., Parameswaran, S. (2017). Secondary vortex-based analysis of flow characteristics and pressure drop in helically coiled pipe. Advances in Mechanical Engineering, vol. 9, no. 4, p. 1-11, DOI:10.1177/1687814017700059.

[6] Sharqawy, M.H., Saad, S.M., Ahmed, K.K. (2019). Effect of flow configuration on the performance of spiral-wound heat exchanger. Applied Thermal Engineering, vol. 161, art. ID 114157, DOI:10.1016/j.applthermaleng.2019.114157.

[7] Patil, R.H. (2019). Fluid flow and heat transfer analogy for laminar and turbulent flow inside spiral tubes. International Journal of Thermal Sciences, vol. 139, p. 362-375, D0I:10.1016/j.jithermalsci.2019.01.036.

[8] Neshat, E., Hossainpour, S. (2017). Numerical investigation of unsteady natural convection heat transfer from the outer surface of helical coils. Proceedings of the Institution of Mechanical Engineers, Part E: Journal of Process Mechanical Engineering, vol. 231, no. 3, p. 383-391, DOl:10.1177/0954408915598130.

[9] Elattar, H.F., Fouda, A., Nada, S.A., Refaey, H.A., Al-Zahrani, A. (2018). Thermal and hydraulic numerical study for a novel multi tubes in tube helically coiled heat exchangers: Effects of operating/geometric parameters. International Journal of Thermal Sciences, vol. 128, p. 70-83, D0l:10.1016/j. ijthermalsci.2018.02.020.
[10] Prabhanjan, D.G., Raghavan, G.S.V., Rennie, T.J. (2002). Comparison of heat transfer rates between a straight tube heat exchanger and a helically coiled heat exchanger. International Communications in Heat and Mass Transfer, vol. 29, no. 2, p. 185-191, D0l:10.1016/S0735-1933(02)00309-3.

[11] Močnik, U., Blagojevič, B., Muhič, S. (2020). Numerical analysis with experimental validation of single-phase fluid flow in a dimple pattern heat exchanger channel, Strojniški vestnik - Journal of Mechanical Engineering, vol. 66, no. 9, p. 10, D0I:10.5545/sv-jme.2020.6776.

[12] Hashemi Karouei, S.H., Mousavi Ajarostaghi, S.S. (2021). Influence of a curved conical turbulator on heat transfer augmentation in a helical double-pipe heat exchanger. Heat Transfer, vol. 50, no. 2, p. 1872-1894, Dol:10.1002/htj.21960.

[13] Sheeba, A., Abhijith, C.M., Prakash, M.J. (2019). Experimental and numerical investigations on the heat transfer and flow characteristics of a helical coil heat exchanger. International Journal of Refrigeration, vol. 99, p. 490-497, D0l:10.1016/j. ijrefrig.2018.12.002.

[14] Jamshidi, N., Farhadi, M., Ganji, D.D., Sedighi, K. (2013). Experimental analysis of heat transfer enhancement in shell and helical tube heat exchangers. Applied Thermal Engineering, vol. 51, no. 1-2, p. 644-652, D0l:10.1016/j. applthermaleng.2012.10.008.

[15] Ravigururajan, T.S., Bergles, A.E: (1996). Development and verification of general correlations for pressure drop and heat transfer in single-phase turbulent flow in enhanced tubes. Experimental Thermal and Fluid Science, vol. 13, no. 1, p. 5570, DOl:10.1016/0894-1777(96)00014-3.

[16] Woods, G.E., Baguley, R.B., Bringas, J.E. (2000). Casti Guidebook to ASME B31.3. McGraw-Hill, New York.

[17] Colebrook, C.F., Blench, T., Chatley, H., Essex, E.H., Finniecome, J.R., Lacey, G., Williamson, J., Macdonald, G.G. (1939). Correspondence. Turbulent flow in pipes, with particular reference to the transition region between the smooth and rough pipe laws.(includes plates). Journal of the Institution of Civil Engineers, vol. 12, no. 8, p. 393-422, Dol:10.1680/ ijoti.1939.14509.

[18] Tang, L., Tang, Y., Parameswaran, S. (2016). A numerical study of flow characteristics in a helical pipe. Advances in Mechanical Engineering, vol. 8, no. 7, p. 1-8, Dol:10.1177/1687814016660242.

[19] Churchill, S.W., SW, C. (1977). Friction-factor equation spans all fluid-flow regimes. Chemical Engineering, vol 84, p. 91-92.

[20] Green, D.W., Perry, R.H. (2008). Perry's Chemical Engineers' Handbook. McGraw-Hill, New York.

[21] Di Piazza, I., Ciofalo, M. (2010). Numerical prediction of turbulent flow and heat transfer in helically coiled pipes. International Journal of Thermal Sciences, vol. 49, no. 4, p. 653-663, Dol:10.1016/j.ijthermalsci.2009.10.001. 